\title{
STRUTTURE PRODUTTIVE E DI SERVIZIO NEI MONASTERI RUPESTRI DELLA CAPPADOCIA. UN'ESPERIENZA RECENTE DI ARCHEOLOGIA "LEGGERA"
}

\author{
FABIO REDI"
}

UDC: 726.71:631(560.4)"05/07"

Preliminary communication

Manuscript received: 30. 08. 2016.

Revised manuscript accepted: 07. 02. 2017.

DOI: 10.1484/J.HAM.5.113729

\author{
F. Redi \\ Dipartimento di Scienze Umane \\ Università degli Studi dell'Aquila \\ Viale Nizza 14, 6710o L'Aquila, Italia \\ fabio.redi@cc.univaq.it
}

\begin{abstract}
A research of "light" archeology conducted in the two year period 2002-2003 in the district of Urgup in Cappadocia has provided some significant information on the internal organization and the territory of the many rock monastic settlements still largely present. According to the recommendations that Girolamo sends to the monk Rustico di Tolosa in 411, the agricultural activity and the necessary channeling of water for irrigation has left signs referable to the monastic presence of VI-VIII century, but also other activities connected with it such as the breeding of pigeons and bees and the cultivation of vines, they have left substantial traces in the rock dwellings of the territory. A final chapter treats the places of the processing and consumption of food of Community.
\end{abstract}

Keywords: Cappadocia, Girolamo, Rustico of Tolosa, monk, monastery, food

Una ricerca di archeologia "leggera” ante litteram, condotta nel biennio 2002-2003 $3^{1}$ e rimasta ancora incompiuta a causa dell'attuale situazione politico-militare nell'area NE della Turchia, nel distretto di Urgup in Cappadocia, ha fornito non pochi spunti di riflessione e dati evidenti sull'organizzazione interna e del territorio dei numerosi insediamenti monastici rupestri che notoriamente ne costellano gli altipiani, le falesie e levallate. Rimandando ad altra sede l'analisi dei luoghi delle celebrazioni liturgiche e delle sepolture, ci soffermeremo in questa occasione sui luoghi della produzione o del lavoro fisico con particolare attenzione ad alcune attività, parte delle quali attestate dalle tradizionali fonti letterarie, altra parte non espressamente ricordata da esse.

La lavorazione e l'irrigazione dei campi e l'allevamento delle api appartengono al primo gruppo di attività, la produzione del vino e l'allevamento dei colombi al secondo.

«Fai cesti con le canne o intreccia canestri con il vimini; zappa la terra e suddividi il tuo orto in piccoli riquadri uguali. E quando avrai piantato gli ortaggi e messo a dimora le piante, porta loro l'acqua attraverso canalette [...]. Innesta fusti infruttiferi con germogli, così che il tuo lavoro possa essere remunerato con i dolci pomi che ne otterrai. Costruisci anche alveari per le api [...] perché tu potrai apprendere da queste piccole creature come organizzare un monastero e come governare un regno. Intreccia corde per farne reti con cui catturare pesci e copia libri. Che le tue mani possano guadagnarti il tuo vitto e la tua mente possa essere soddisfatta nella lettura» ${ }^{2}$. Tutto questo Girolamo raccomandava al monaco Rustico di Tolosa in una lettera del 411, riferendosi evidentemente a esempi dell'arco orientale del Mediterraneo, almeno dall'Egitto alla Siria, se non fino all'Anatolia.

Sicuramente l'aspetto preponderante espresso dalla lettera di Girolamo è costituito dall'attività agricola, ortiva in particolare se praticata all'interno del recinto claustrale, con una nota specifica riguardante la geometrizzazione degli spazi ortivi, l'innesto degli alberi da frutto, ma anche l'indispensabile canalizzazione dell'acqua per l'irrigazione. L'allevamento delle api è spunto di riflessione e di apprendimento di come organizzare gli spazi e la vita sociale, cioè di ciò che attiene all'urbanistica e alla politica. Altre attività manuali riguardano la produzione di cesti o di canestri di vimini e di corde per le reti da pesca, mentre solo un breve cenno, «copia libri», è rivolto all'attività amanuense degli scriptoria.

\section{ORGANIZZAZIONE DEI TERRENI AGRICOLI E IRRIGAZIONE}

Se nel territorio esaminato, trattandosi di terreni agricoli ricavati nelle vallate irrigue, all'esterno degli spazi monastici, non possiamo riconoscere $\mathrm{i}$ «piccoli riquadri» dei quali parla Girolamo nella lettera citata, sono frequenti però alcune opere di terrazzamento o di arginatura per trattenere l'acqua di irrigazione dei campi e impedire l'erosione dei terreni agricoli da parte delle acque meteoriche, quando particolarmente abbondanti, ed è significativo come le opere di canalizzazione siano attuate secondo precisi criteri di economia idrica e di suolo, attraverso sifoni, inghiottitoi e percorsi sotterranei che bypassano ove necessario i terreni coltivati. Ci troviamo, infatti, su altipiani o in valli che si attestano intorno ai $1000 \mathrm{~m}$ di quota, soggette a forti nevi-

\footnotetext{
* Le illustrazioni prive di rimando ad autori o testi sono tutte opera di F. Redi.

${ }^{1}$ E. BURRI, C. GERMANI, C. MALANDRA, F. REDI, L'insediamento sotterraneo di Karlik in Cappadocia (Distretto di Urgup - Provincia di Nevsehir - Turchia), in Archeologia medievale, 29, 2002, p. 355-369; E. BURRI, A. DEL BON, C. IOVENITTI, F. REDI, La Meskendir Vadisi e la cultura materiale delle aree limitrofe (Cappadocia - Turchia), in Archeologia medievale, 30, 2003, p. 453-481.

${ }^{2}$ HYERONIMUS, Epistulae I-CLIV, 3 voll., a cura di I. Hilberg, M. Kamptner, Wien, 1910, 1918, 1996 (Corpus scriptorum ecclesiasticorum latinorum, 54, 55, 56 1/2), CXXV, 11; F. MARAZZI, I luoghi della produzione artigianale nei monasteri altomedievali europei. Un excursus sulla base delle fonti scritte e archeologiche, in Teoria e pratica del lavoro del monachesimo altomedievale, Atti del Convegno internazionale di studio (Roma - Subiaco, 7-9 giugno 2013), Spoleto, 2015 (De re monastica, IV), p. 232.
} 


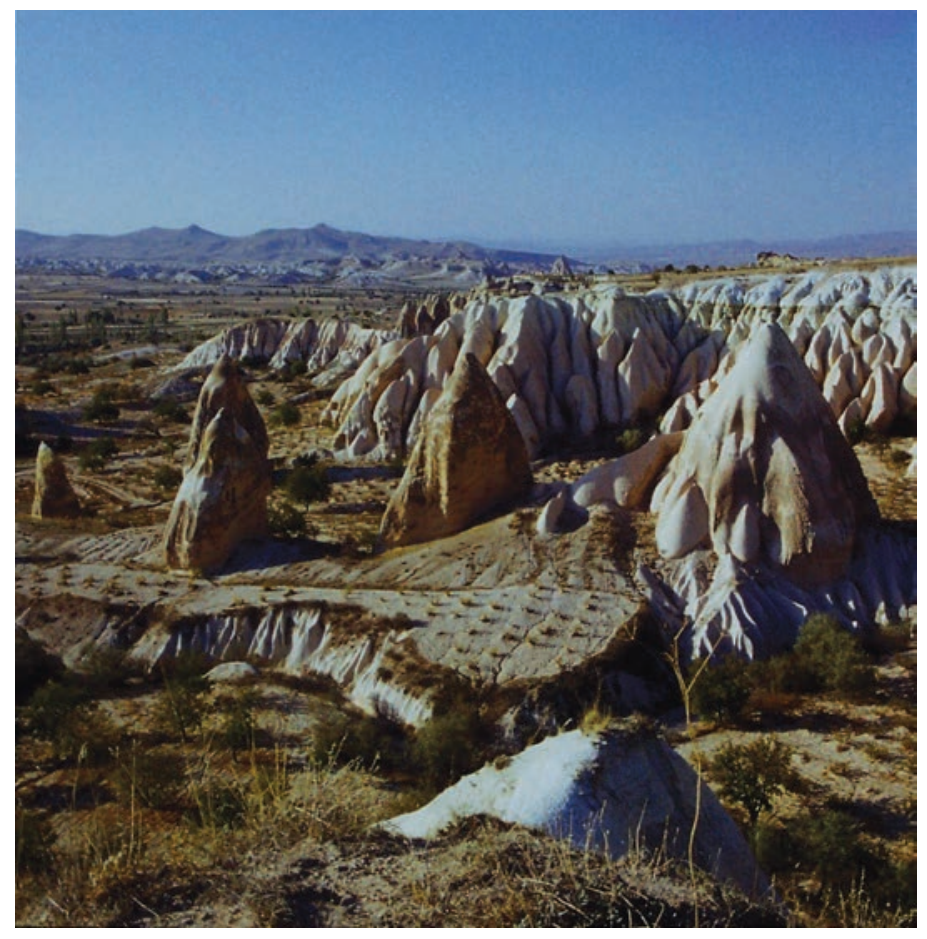

Fig. 1. Paesaggi agricoli della Meskendir Vadisi.

cate invernali e a siccità ed elevate temperature estive, con escursioni termiche stagionali e diurne piuttosto sensibili³

Pedologicamente i terreni sono fertili, in quanto di origine vulcanica (ignimbriti) e friabili, sciolti, nelle vallate, anche oggi ricche di alberi da frutto (meli, albicocchi e viti in particolare) e destinate a seminativo o a ortaggi (cavoli, legumi, peperoni, carciofi, cucurbitacee) (fig. 1). Le canalizzazioni dell'acqua sono praticate marginalmente

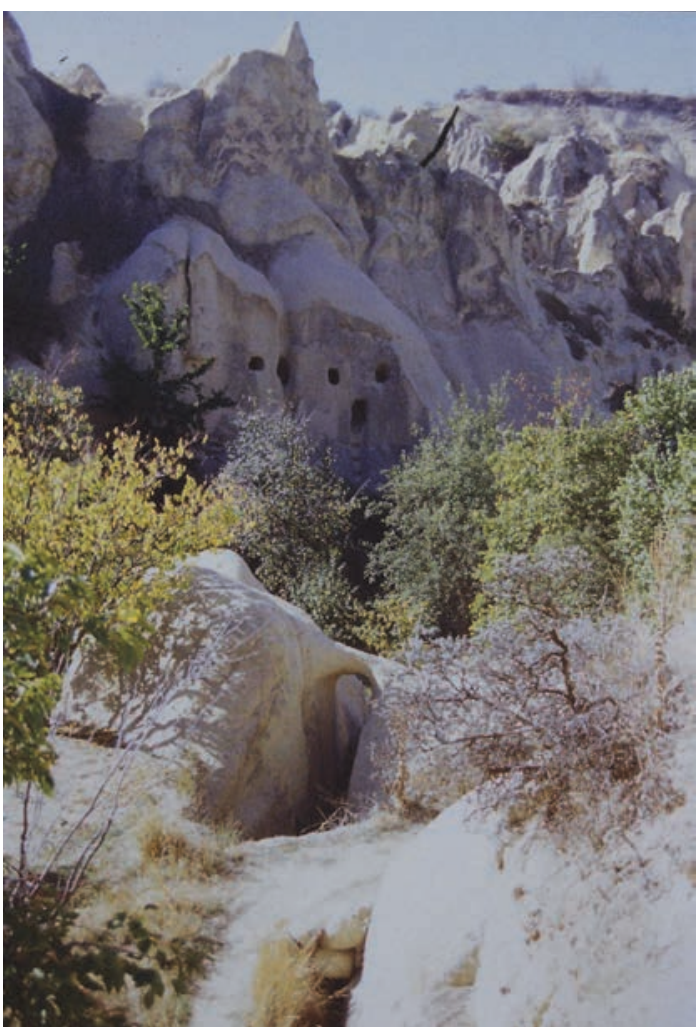

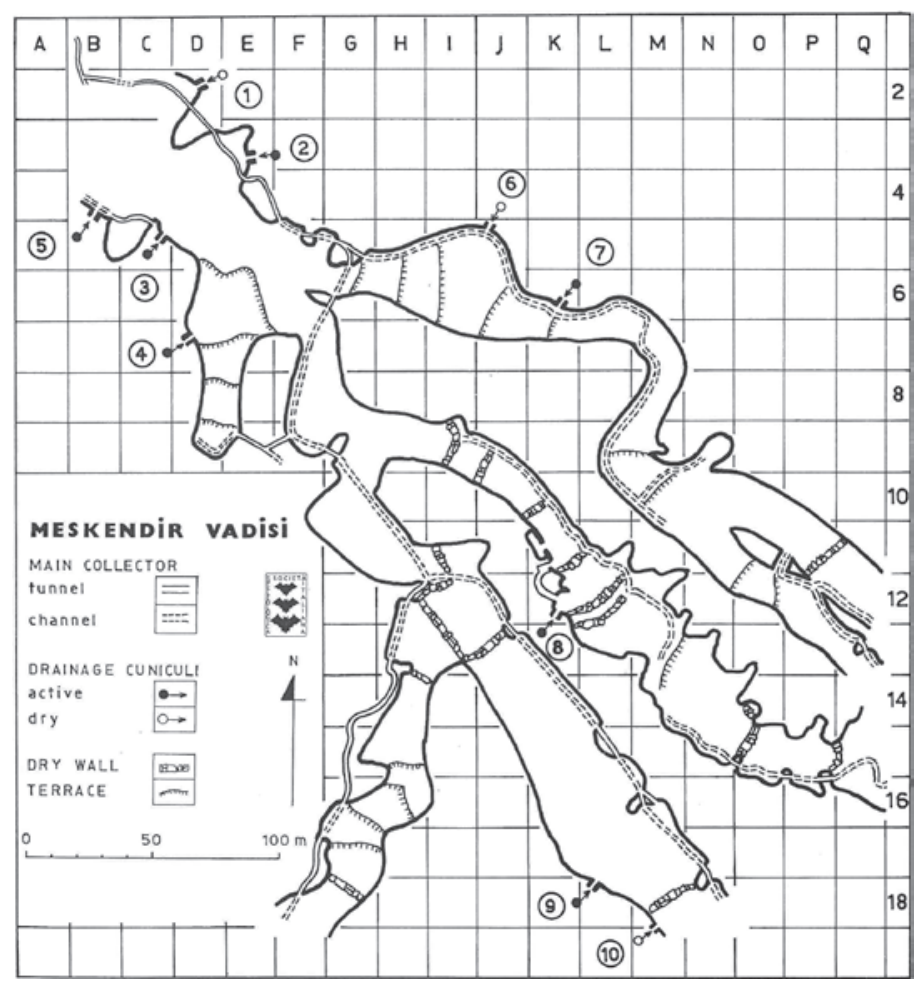

Fig. 2. Organizzazione dei terreni agricoli e irrigazione [Carta della Meskendir Vadisi, da A.R. Bicchi, E. Burri, M. Castellani, V. Castellani, G. Pensabene, op. cit. (n. 3), p. 81, fig. 2].

rispetto agli appezzamenti agricoli, così da non sottrarre terreno utile per le colture e da non asportarlo nei periodi di piena. Frequentemente esse sono incassate nella roccia ignimbritica o arginate se superficiali; possono presentare derivazioni all'inizio del terrazzo agricolo e recuperi alla

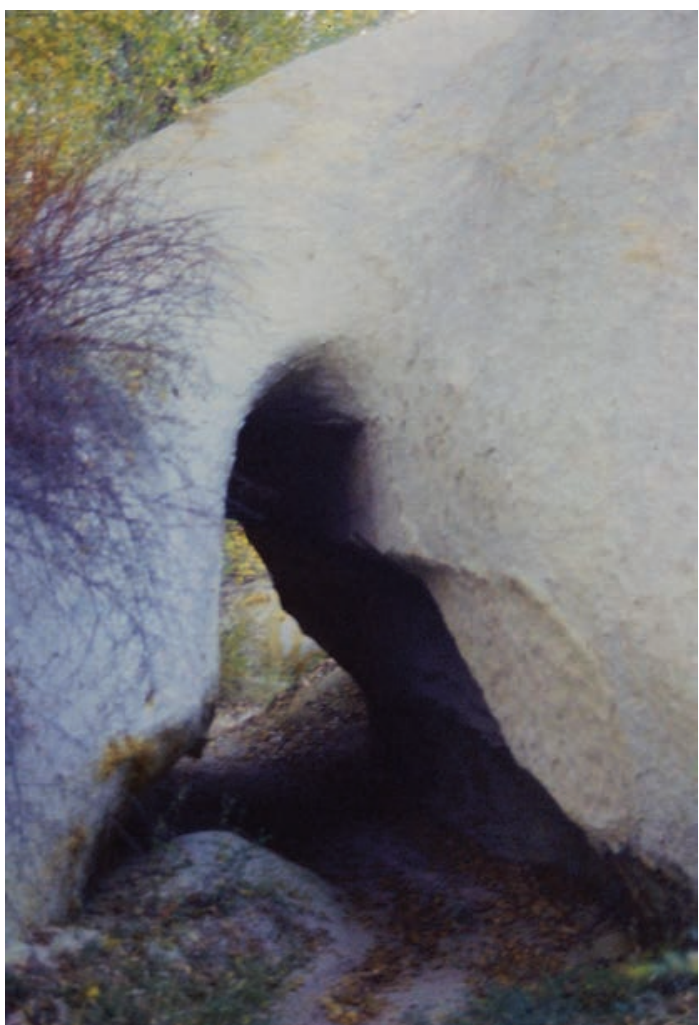

Figg. 3-4. Tracce di opere idrauliche della Meskendir Vadisi.

${ }^{3}$ A.R. BICCHI, E. BURRI, M. CASTELLANI, V. CASTELLANI, G. PENSABENE, Evidences for hydrogeological planning in ancient Cappadocia, in Le città sotterranee della Cappadocia, Opera ipogea, supplemento a Speleologia, 1, ottobre 1995, p. 78-86; R. BIXIO, V. CASTELLANI, Tipologia delle strutture sotterranee della Cappadocia, in Le città sotterranee, cit. supra, p. 118-120; E. BURRI, L'antico sistema di gestione delle acque nella Meskendir Vadisi (Distretto di Urgup - Provincia di Nevsehir - Turchia), in Archeologia medievale, 29, 2002, p. 370-377. 


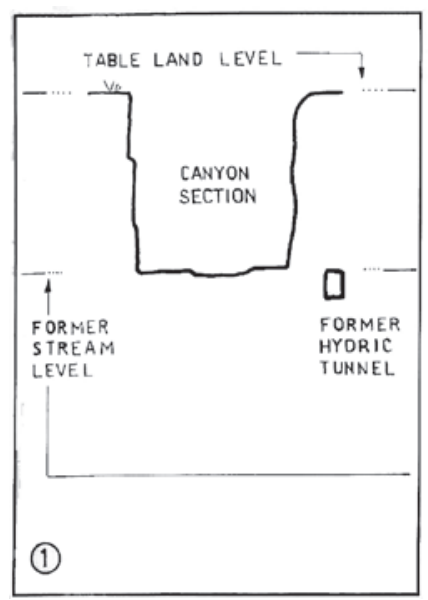

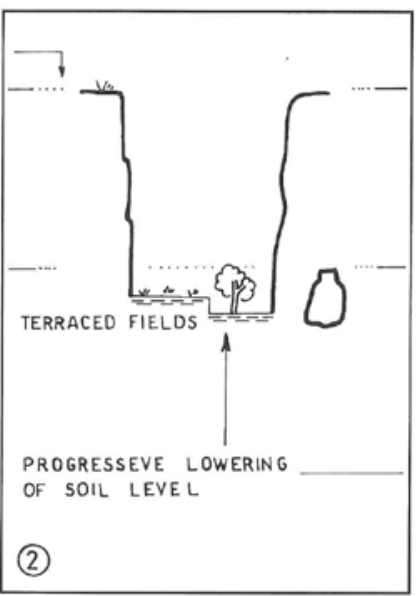

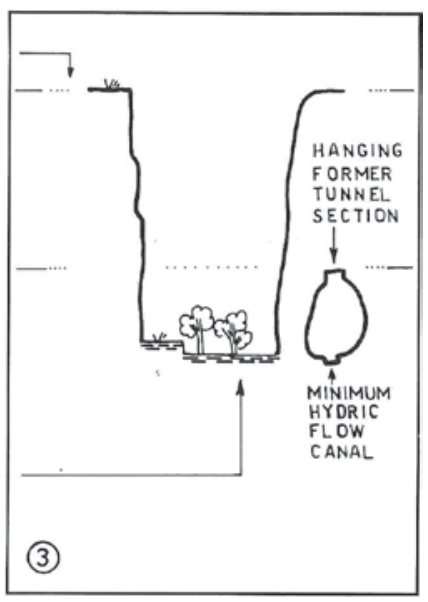

Fig. 5. Schema evolutivo delle canalizzazioni e della morfologia di una valle [da A.R. Bicchi, E. Burri, M. Castellani, V. Castellani, G. Pensabene, op. cit. (n. 3), p. 85, fig. 5]. che fungono da conserva dell'acqua o da trasmissione a terrazzi agricoli più distaccati e a quote molto inferiori (figg. 3-4). Interessante è l'osservazione delle modalità e delle fasi di escavazione delle condutture idriche, ben riconoscibili dai segni del picconcello usato dai fossores nel procedere dello scavo e dell'allacciamento fra condutture o percorsi diversi, ma anche e soprattutto dalla forte erosione che nel tempo le canalizzazioni hanno subito modificando ampiezza e forma delle loro sezioni trasversali (fig. 5).

\section{ALLEVAMENTO DEI COLOMBI E PRODUZIONE DI CONCIME}

fine, oppure percorsi a zig-zag che iniziano lungo il capo del campo, proseguono perimetralmente e scendono al terrazzo successivo tramite un piccolo salto al piede del terrazzo (fig. 2). Sono frequenti percorsi idrici sotterranei o "di caduta" entro inghiottitoi, talvolta naturali, altrimenti artificiali,

Strettamente collegata con le coltivazioni agricole, sia pure come effetto secondario dell'allevamento dei colombi per uso alimentare, è l'abbondante produzione di guano per la concimazione. Il territorio è densamente interessato
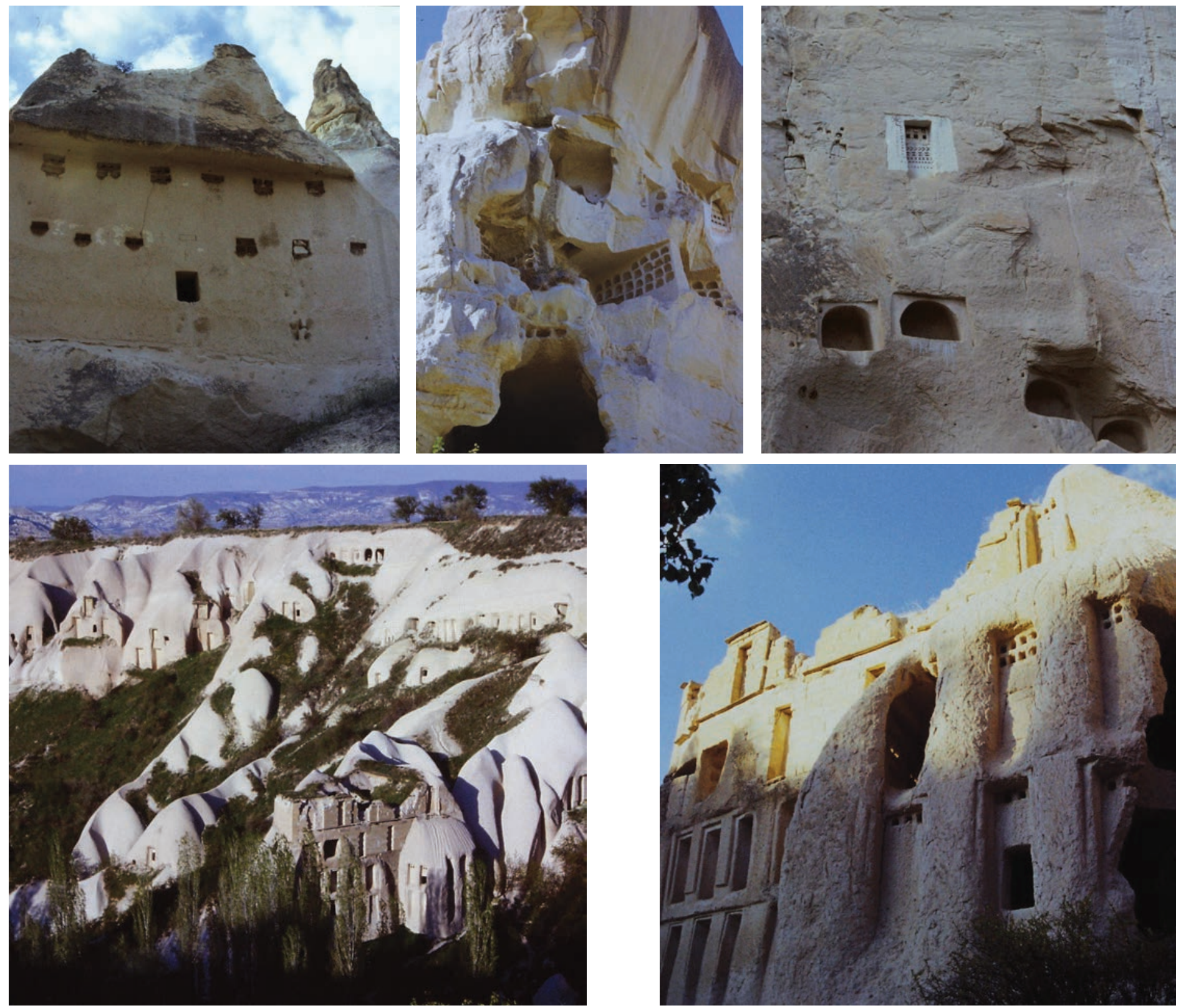

Figg. 6-10. Esempi di colombaie nelle valli di Göreme, Meskendir, Kizil Çukur. 
da colombaie, tanto che a Uçhisar un'intera vallata prende il nome di "Valle dei Piccioni". Le colombaie risultano per lo più inserite come uso secondario in strutture precedentemente destinate ad altre funzioni, come ossari di cimiteri rupestri, o come abitazioni troglodite 4 . Nel primo caso il reimpiego delle piccole celle quadrangolari, o stondate, scavate nella roccia ignimbritica secondo allineamenti orizzontali e verticali regolari per il contenimento dei crani ed eventualmente di ossa lunghe in una fase di riduzione scheletrica per sepolture secondarie, non presenta un particolare e percepibile intervento di adattamento o modificazione delle preesistenze. Nel secondo caso, l'escavazione delle celle quadrangolari o di particolari alloggiamenti, raramente a sezione triangolare, interferisce evidentemente con le strutture preesistenti, intervenendo ex novo, su superfici piane o interfacciando e tagliando precedenti manufatti con destinazioni d'uso diverse.

Generalmente si preferiva situare le colombaie in strutture impervie e sensibilmente rilevate da terra o in siti appartati e difficilmente accessibili, come nelle valli di Meskendir, Güllü Dere, Kizil Çukur, ecc. (figg. 6-10). Una particina in quota, anche a qualche decina di metri da terra, consentiva l'accesso dell'allevatore entro vani di modesta cubatura, monovolumi o con più camere comunicanti, opportunamente provvisti di celle quadrangolari allineate fra loro, che veniva murata, per impedire il furto dei colombi, risparmiando nella porta-parete una o più piccole aperture per consentire l'accesso e l'uscita dei colombi. Attorno a queste aperture rimangono ancora leggibili motivi apotropaici assai vari e iscrizioni di diversa consistenza dipinti a sanguigna (la città di Sinope non è eccessivamente distante dalla Cappadocia) o in policromia con pigmenti di colore bruno od ocra a base di calce (fig. 11).

Un esemplare significativo rimane nella media Valle di Göreme come probabile utilizzo secondario di strutture funerarie. Ogni cella-ossario è sottolineata da un contorno a sanguigna e una cella più ampia, a rettangolo trasversale, è inquadrata da un semicerchio con croce sul culmine ed ele-

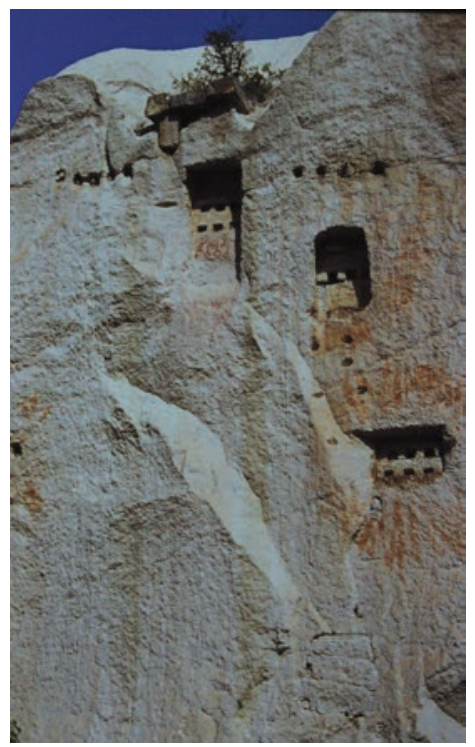

Fig. 11. Pitture di una colombaia nella Fig. 12. Pitture di una colombaia nella Meskendir Vadisi.

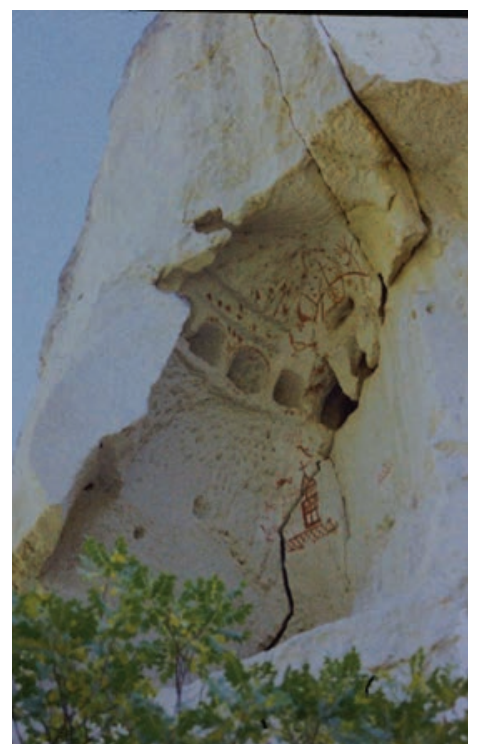

valle di Göreme. menti vegetali. Sono raffigurati anche, di profilo, due grossi colombi e uno sciame di piccoli piccioni tutto attorno, come più in basso, attorno a un campanile o torre piccionaia, con croce sulla cuspide (fig. 12). Del resto, non sembra eccessivo accostare la raffigurazione del volo dei colombi che si librano dalle celle-ossario all'immagine delle anime che si separano dal corpo con la morte, in una sorta di continuità semantica fra la funzione primitiva e quella secondaria delle cavità in esame. A Uçhisar la Valle dei Piccioni presenta edifici rupestri con facciate monumentali, in origine addetti al culto dei morti, con ossari molto voluminosi trasformati in colombaie.

Quasi sempre la porticina in quota è preceduta sulla verticale da "pedarole", generalmente doppie, parallele ma sfalsate, o singole in successione verticale, per consentire all'allevatore l'accesso alla colombaia con l'ausilio di una lunga fune di sostegno, assicurata a un anello ricavato a fianco della porticina e/o ai piedi della "pedarola" inferiore, sia pure in quota per rendere inaccessibile la colombaia. In assenza delle "pedarole" l'accesso poteva avvenire con lunghe scale a braccioli di legno o semplicemente "a braccia", tramite una fune, puntando i piedi sul costone di roccia verticale (figg. 13-14). L'occlusione in muratura dell'accesso alla colombaia sembra funzionale alla raccolta del guano, che richiede tempi alquanto dilazionati, piuttosto che alla più frequente cattura dei colombi per l'alimentazione. Essa perciò sembra essere stata limitata solo agli individui adulti e avvenire fuori dalla colombaia con l'uso di reti.

\section{ALLEVAMENTO DELLE API E PRODUZIONE DI MIELE}

L'invito a costruire alveari per le api con lo scopo materiale o pratico della raccolta del miele per la sussistenza dei monaci o della cera per l'illuminazione, ma anche, e forse principalmente, per l'intento morale di apprendere da queste piccole creature la capacità di organizzazione gerarchica degli spazi sia monastici sia urbani e di governo di una comunità, espresso nella già ricordata lettera di Girolamo, trova attuazione nei numerosi esempi che abbiamo riscontrato
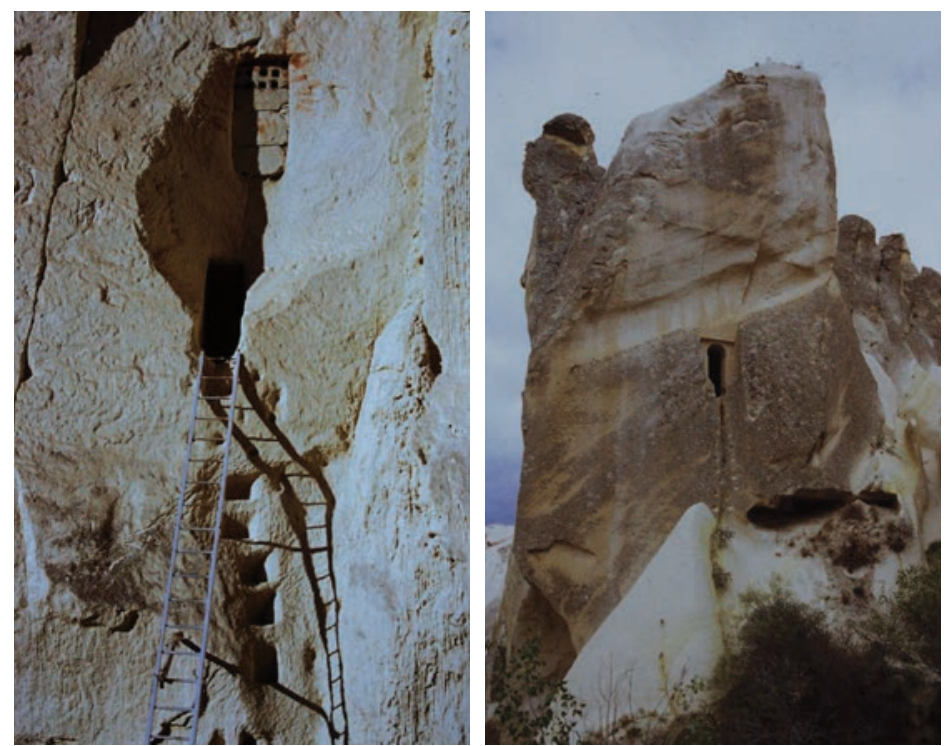

Figg. 13-14. Esempi di colombaie con "pedarole": valli di Kizil Çukur e Meskendir.

${ }^{4}$ R. BIXIO, V. CASTELLANI, Tipologia delle strutture sotterranee della Cappadocia, in Le città sotterranee, op. cit. (n. 3), pp. 110-113; F. REDI, Insediamenti rupestri della Cappadocia: considerazioni preliminari e problemi di metodo. Prime acquisizioni, in Archeologia medievale, 30, 2003 , p. 475-477. 

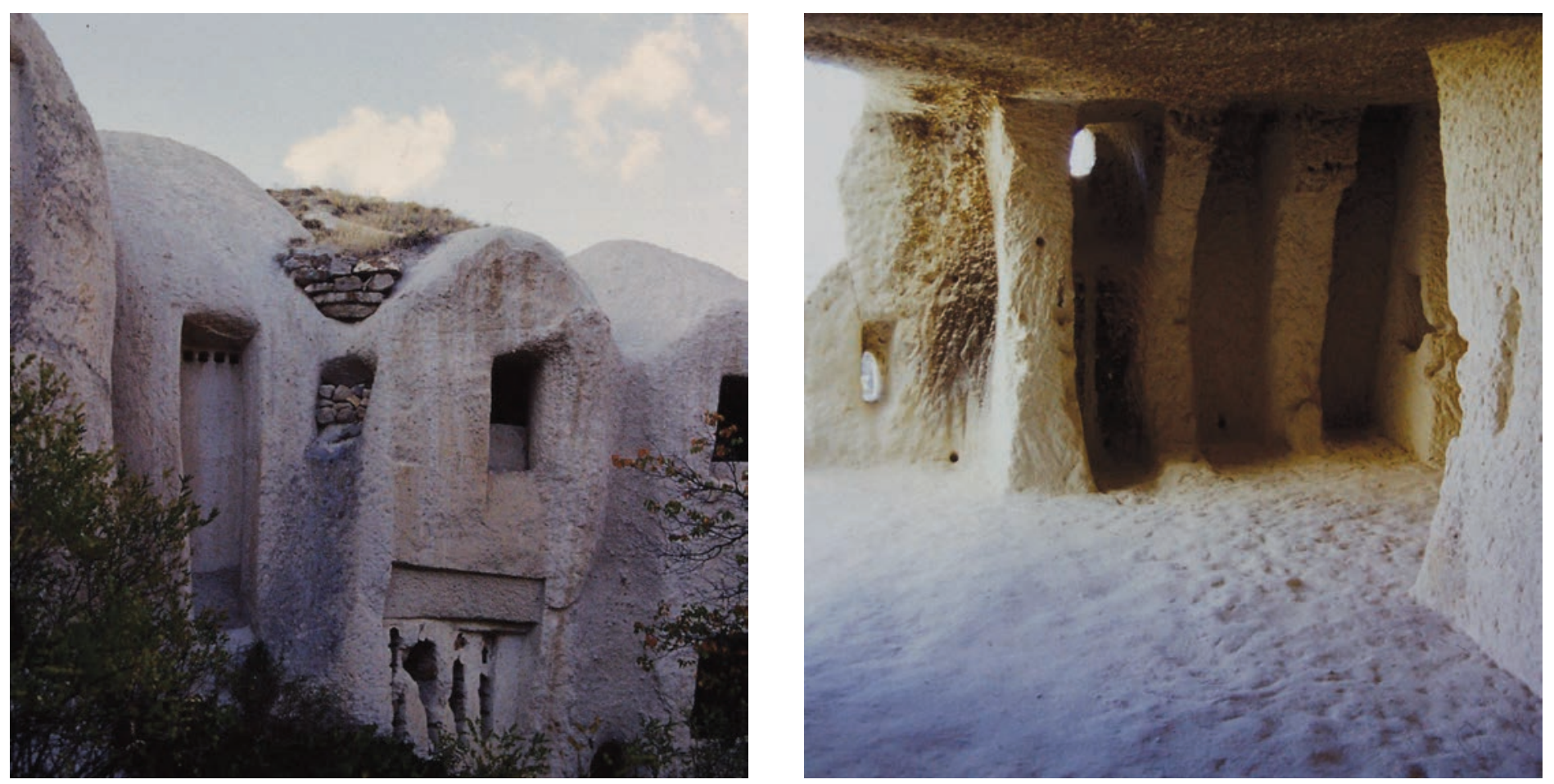

Figg. 15- 16. Apiari della Meskendir Vadisi e della Aynali Kilisi.

nel territorio esaminato, come nelle valli di Meskendir, Kizil Çukur, Göreme, ecc.5 (figg. 15-16).

In prossimità dei monasteri, ma anche distaccati nel territorio, permangono ambienti con caratteristiche particolari, di diversa consistenza, ma con forme ben definite e standardizzate, funzionali all'allevamento delle api. Elementi connotativi costantemente presenti sono alcune file verticali e parallele di piccoli fori circolari equidistanti, frequentemente provvisti di "posatoio" esterno per le api, con abbondanti colature di miele e/o di cera ancora evidenti, definiti "fori di volo", di numero variabile da 12 a 20, organizzati in orizzonti di 3 o di 5 , ripetuti verticalmente 4 o 5 volte. I "fori di volo" erano praticati trapanando la parete rocciosa, frequentemente sbassata in piano, come negli apiari della Aynali Kilisi e di Kizil Çukur. Generalmente si riscontra anche una serie di sottili, lunghe e ravvicinate feritoie di areazione, in numero variabile da 2 a 5, separata dai "fori di volo" tramite l'interposizione di una porticina di accesso agli ambienti interni.

Entro quello principale, ai "fori di volo" corrisponde una struttura particolare, costituita da nicchie verticali, di forma rettangolare allungata, profonde mediamente $50 \mathrm{~cm}$, separate da pilastri a sezione rettangolare aggettanti quanto la profondità delle nicchie, in numero uguale a quello delle serie verticale dei "fori di volo". Dette nicchie, erroneamente interpretate da alcuni come latrine ${ }^{6}$, erano funzionali al contenimento delle arnie, separate da ripiani di legno talvolta inseriti in apposite scanalature orizzontali, come quelle nell'apiario della valle di Kizil Çukur, e chiuse verso l'interno della struttura per mezzo di tavole, amovibili per la raccolta del miele o della cera. Tenuto conto del numero sostenibile di alveari per ettaro, coltivato a meli o albicocchi, in rapporto di 3-5 arnie in gruppi di $4-6$, si può calcolare che apiari di circa 20 arnie sono compatibili con la scala di grandezza degli esemplari rinvenuti.

Meno definibile è la funzione delle serie di feritoie che affiancano la porta di accesso all'apiario e che sicuramente contribuiscono alla ventilazione degli ambienti, in particolare di quello corrispondente, nel quale si ritiene che risiedesse l'apicoltore nei periodo di raccolta del miele e della cera, o più probabilmente ritengo venisse conservato il fieno raccolto, qualificando quindi queste particolari strutture come soluzioni polifunzionali integrate, come si verifica attualmente in una struttura superstite nell'alta valle di Göreme.

Oltre ad apiari caratterizzati dalle strutture ora presentate abbiamo riscontrato anche altre forme, come nell'apiario della Aynali Kilisi di Firkatan. Insieme con la struttura a celle verticali separate da pilastrini di roccia risparmiati dall'escavazione è presente nello stesso ambiente un sistema costituito da arnie di legno pensili, poggianti su mensole e tavola lignee, delle quali rimangono soltanto gli alloggi quadrati, disposti in orizzontale e ravvicinati, nella parte alta della stanza, abbinati a rari "fori di volo" verticali, che fuoriescono dal soffitto dell'apiario.

\section{VITICULTURA E PRODUZIONE DI VINO}

Nonostante la già citata lettera di Girolamo non faccia riferimento esplicito alla viticoltura e alla produzione di vino, è molto probabile che le numerose tinaie e vasche da spremitura dell'uva presenti nel territorio in esame possano riferirsi al periodo dei monasteri bizantini piuttosto che a quello successivo islamico ${ }^{7}$. Nonostante il divieto coranico,

${ }_{5}$ F. REDI, op. cit. (n. 4), p. 474; R. BIXIO, F. DAL CIN, M. TRAVERSO, Cappadocia: un apiario rupestre. Un nuovo tipo di cavità artificiale nella valle di Kizil Çukur (Turchia), in Opera Ipogea, 2, 2002, p. 17-28.

${ }^{6}$ G.R. ELFORD, La Cappadocia e le città segrete, Fiesole (Fi), 1992, p. 54-55.

7 F. REDI, op. cit. (n. 4), p. 347. 

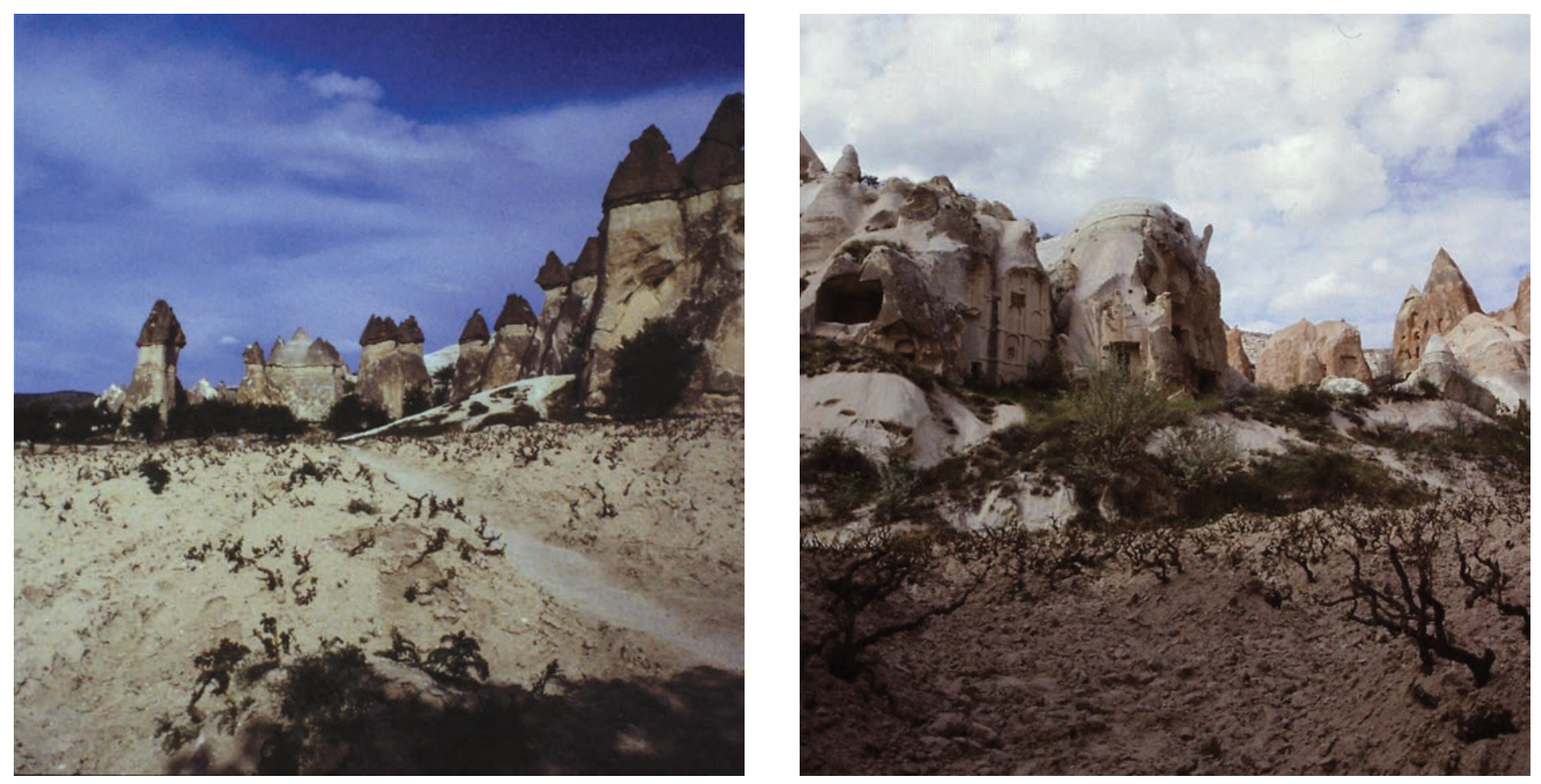

Figg. 17-18. Vigneti delle valli di Paşa Bầ e Güllü Dere.

però, ancora oggi sono numerosi i vigneti attivi, che testimoniano certamente, con l'elevato quantitativo di produzione di vino, non soltanto la pratica dell'esportazione (figg. 17-18).

Sta di fatto che le strutture produttive di vino si trovano prevalentemente annesse ai monasteri e alle chiese di età bizantina, come a Karlik, alla Aynali Kilisi di Ferkatan, nelle valli di Kizil Çukur, di Güllü Dere, di Zelve (figg. 19-21), o distanti dagli insediamenti sia monastici sia laici e isolate nella campagna, come nell'alta valle di Meskendir (fig. 22). L'arco cronologico al quale attribuire le strutture per la produzione di vino presenti nel territorio, non definibile con precisione a causa dei noti limiti di datazione delle strutture rupestri, in quanto realizzate in negativo, è comunque molto ampio. Un esempio significativo abbiamo rinvenuto nella valle di Güllü Dere, nel quale la porta di accesso a una tinaia, esistente all'interno di un monastero, taglia l'arcosolio di tombe scavate nella fase originale di vita dello stesso, in una cripta sepolcrale attigua alla tinaia (fig. 23). Essa tuttavia presenta accesso originale indipendente dalla camera sepolcrale e la porta che taglia l'arcosolio risulta secondaria anche alla fase di attività della tinaia in quanto la battuta della porta stessa cade in parte nella vasca per la spremitura dell'uva e sopra il cordolo di delimitazione dell'invaso di essa, che risulta opportunamente sbassato quasi a raso per l'occorrenza.

Ancora oggi i vigneti dei fertili terreni ignimbritici del territorio presentano impianti tradizionali caratterizzati da viti ad alberello, con chioma appiattita al suolo, dal quale dista circa mezzo metro, per difendere la pianta dai rigori invernali e trattenere il più possibile gli irraggiamenti di calore emanati a riverbero dal terreno (fig. 24).

Gli ambienti per la produzione del vino sono costituiti da un volume, o due come a Karlik e nell'alta Meskendir Vadisi, per la spremitura dell'uva, la raccolta del mosto e la fermentazione, il primo ed essenziale; il secondo, ove esistente, era addetto alla conservazione del vino in contenitori amovibili, non pervenuti, ma attestati da pancali di roccia risparmiati lungo il perimetro degli ambienti. A Karlik uno dei pancali presenta al centro un'ampia cavità rotonda, che si restringe
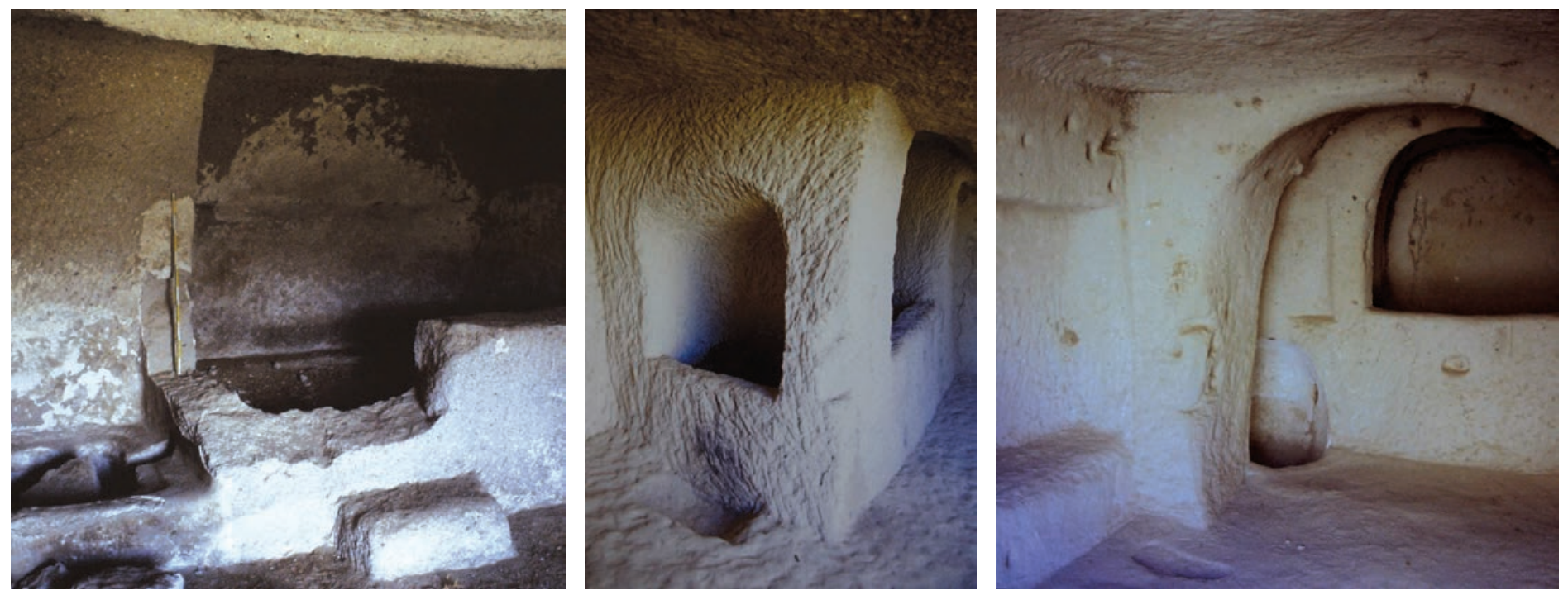

Figg. 19-20-21. Tinaie per la spremitura delliva a Karlik, Aynali Kilisi e Zelwe. 


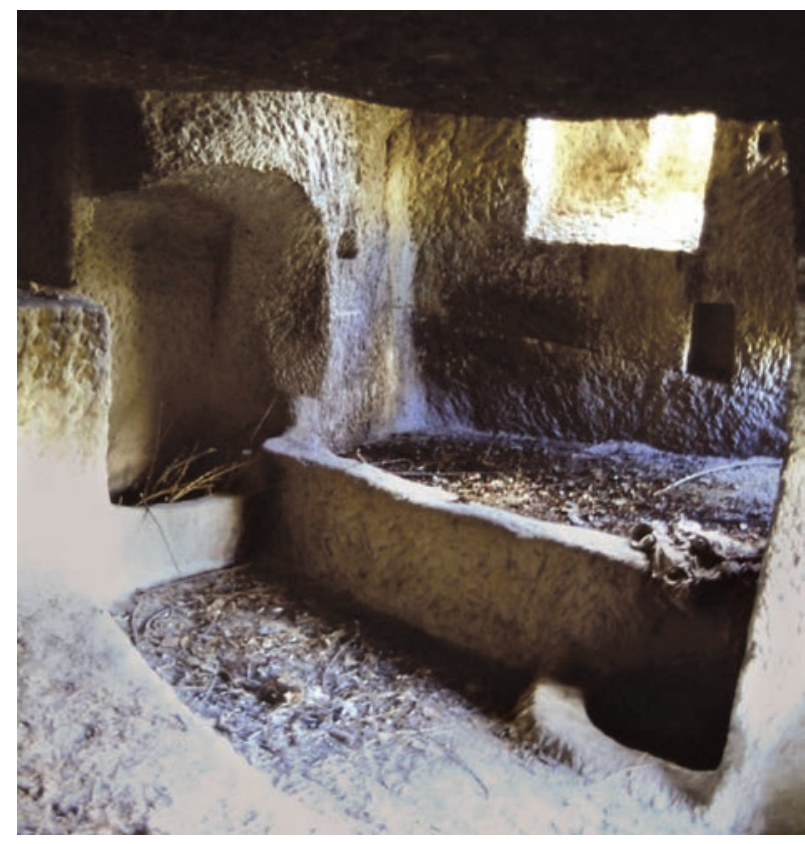

Fig. 22. Tinaia della Meskendir Vadisi.

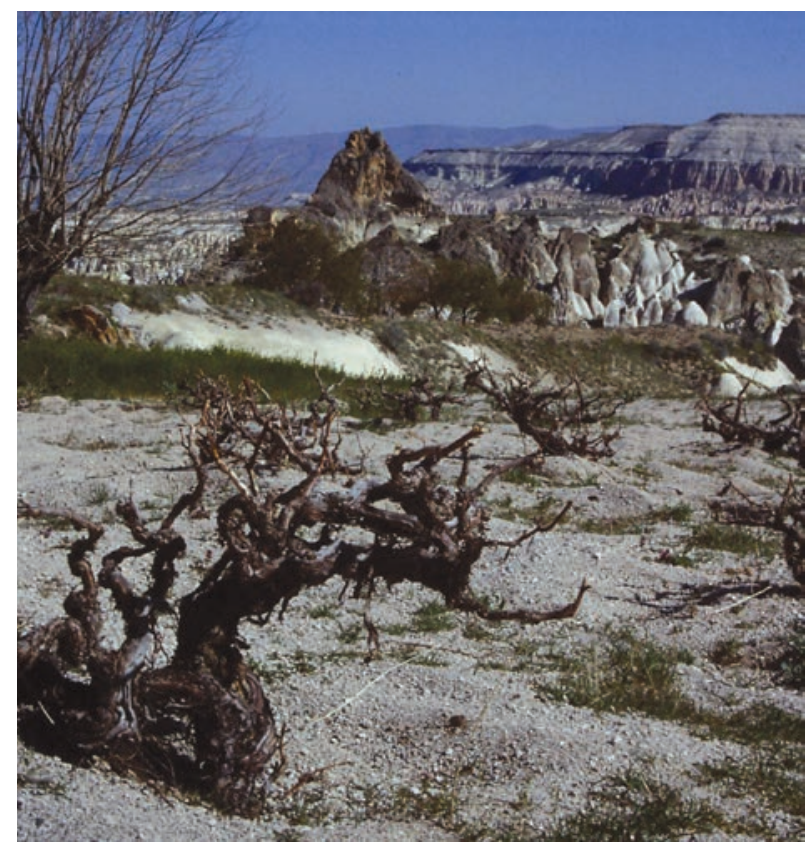

Fig. 24. Particolare coltura di vite nella Meskendir Vadisi.

con un gradino a circa $30 \mathrm{~cm}$ dal bordo, probabilmente per l'inserimento di un grosso contenitore metallico sotto il quale accendere il fuoco, alimentato da una piccola bocca dall'esterno, a lato della cavità rettangolare scavata al centro dell'ambiente per il calpestio degli addetti (fig. 25). Se in fase originale coeva della realizzazione dei tre pancali perimetrali, la struttura circolare ora detta potrebbe sottendere la pratica della cottura del mosto o del vino.

Esistono esempi di minore capienza, come nelle valli di Zelve, Güllü Dere, Kizil Çukur, Meskendir, ecc. e nel monastero di San Sebastiano, nei quali tinaia e cantina sono concentrati in un solo ambiente di dimensioni più ampie, o come nella Aynali Kilisi di Ferkatan nella quale la tinaia e l'apiario sembrano convivere fin dalle origini dell'ambiente e il tino assume forme monumentali "a baldacchino". Gli accessi, tramite porticine rettangolari, per ovvia comodità di esercizio, non erano situati in quota, come per le colombaie e per gli apiari, bensì preceduti soltanto da uno o due gradini.

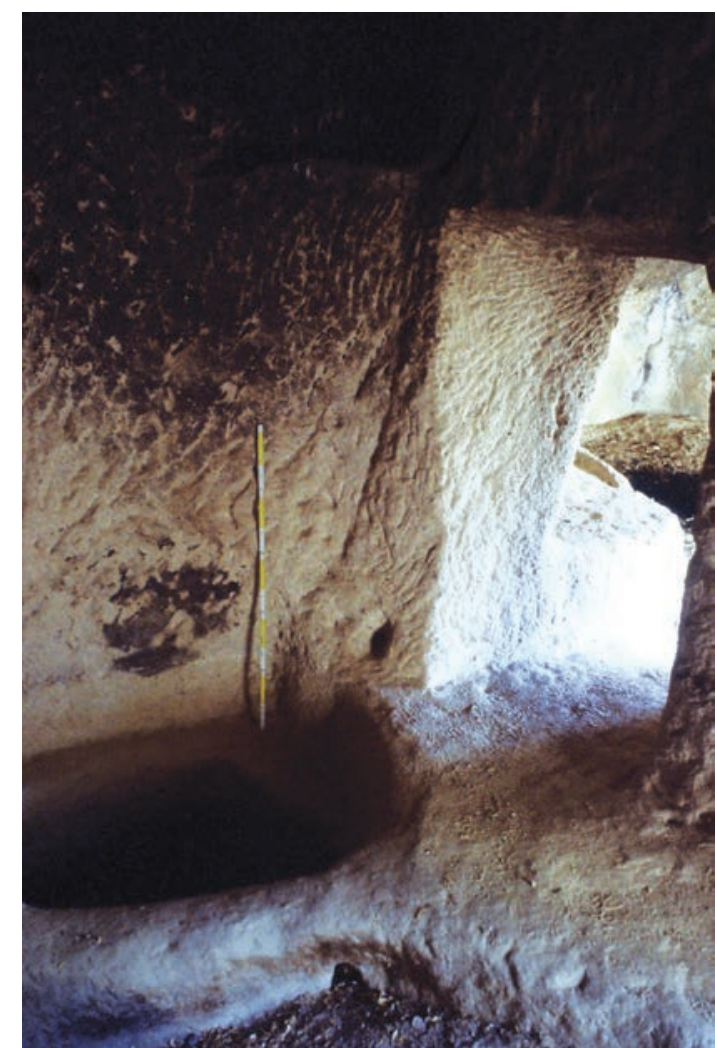

Fig. 23. Tinaia della valle di Güllü Dere.

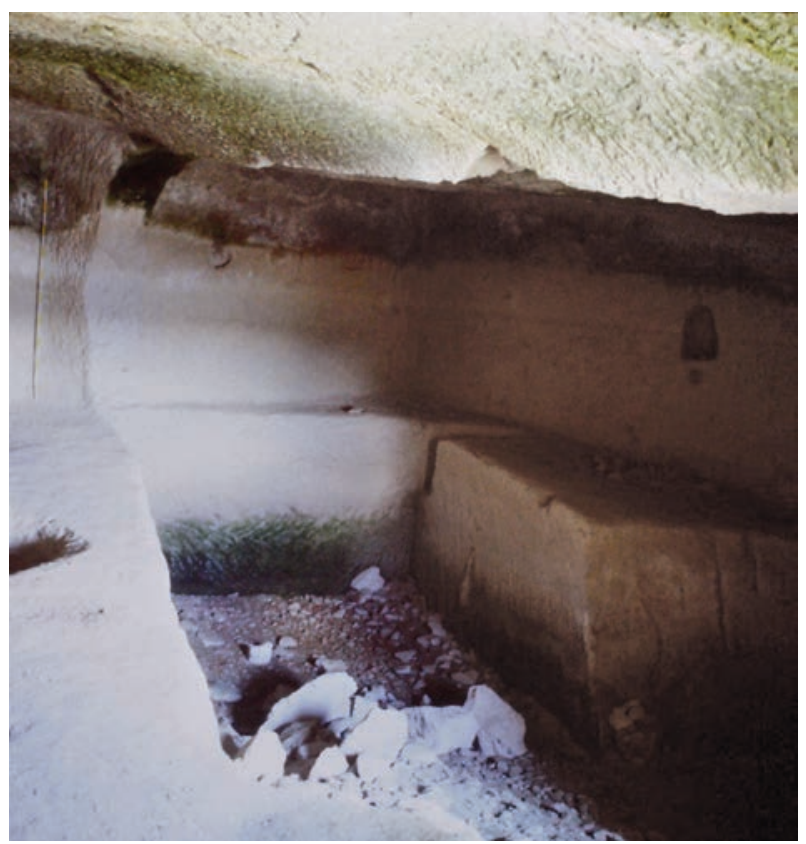

Fig. 25. Cantina con banconi per botti e fornello a Karlik.

L'elemento principale della struttura produttiva consiste in una vasca rettangolare, di ampiezza variabile, profonda non più di 1,20 $\mathrm{m}$, delimitata da pareti spesse mediamente $25 \mathrm{~cm}$, che comunica con una vaschetta di raccolta del liquido, di minori dimensioni e situata a un livello più basso, tramite un foro con piccola doccia. Non essendo state rinvenute nelle pareti o nel pavimento e nel soffitto cavità per l'inserimento di travature di sostegno delle componenti del torchio, riteniamo plausibile che la spremitura dell'uva avvenisse mediante pestatura con i piedi o con magli a mano entro le vasche stesse. Abbiamo riscontrato vaschette di raccolta di due tipi: poco profonde (non più di $60 \mathrm{~cm}$ ) e con lati di piccola ampiezza (meno di $1 \mathrm{~m}$ ), e a pozzo, di mag- 
giore profondità e ampiezza. Nelle prime è probabile che la raccolta del mosto o del vino avvenisse direttamente entro contenitori (botticelle o anforacei) alloggiati nelle vaschette stesse che servivano da raccolta delle eccedenze versate, o in alternativa vi si attingesse il liquido con un romaiolo o con una brocchetta per inserirlo, di seguito, nel contenitore per la conservazione.

Nelle seconde si lasciava versare dalla vasca di spremitura il liquido per poi raccoglierlo con un romaiolo o con una brocchetta per imbottarlo o invasarlo.

Come abbiamo detto, non avendo rinvenuto in nessun caso elementi di copertura delle vasche di spremitura salvo alcuni incavi che ne potrebbero attestare la presenza, la fermentazione poteva avvenire in un altro locale e in altri contenitori, peraltro non rinvenuti, ma possiamo ritenere, in alternativa, che essa avvenisse nelle vasche stesse, essendo sufficiente sigillare la porta di accesso all'ambiente, se di modeste dimensioni. Altrimenti, con una serie di tegoloni o di lastre, che potevano essere murati dopo l'inserimento entro le scanalature già dette, ove presenti, venivano sigillate le vasche di spremitura contenenti il mosto.

\section{CUCINE E REFETTORI: FORNI, FORNELLI, ACQUAI, DISPENSE}

I complessi monastici del territorio offrono esempi significativi di ambienti destinati alla elaborazione e cottura dei cibi e al loro consumo comunitario. In generale le cucine, distinte dai refettori ma prossime a essi, sono costituite da ampi ambienti quadrangolari nei quali spiccano uno o più fornelli scavati nel pavimento e una vasca per l'acqua a pozzo o a cassone ${ }^{8}$. Nelle pareti fortemente annerite dal fumo sono scavate nicchie di diversa forma e collocazione destinate ad acquaio, a ripostiglio per cibi e oggetti d'uso, a contenere lanterne per l'illuminazione.

I fornelli, scavati nel terreno con una profondità di 40-70 $\mathrm{cm}$, hanno forma circolare di diversa ampiezza e sono associati in ogni caso a canalette a sezione quadrangolare, inclinate verso il fornello e direzionate verso la porta di accesso così da ricevere aria per il tiraggio dei carboni che venivano inseriti nella cavità del fornello, sotto pentole o paioli (figg. 26-27). Nell'alta valle di Mekendir si rinvengono casi di incamiciatura delle pareti del fornello con terra refrattaria.

Nel monastero degli Arcangeli a Cemilköy, oltre ad alcuni fornelli scavati nel pavimento, troviamo una parete attrezzata con grande vasca a cassone scavata nella roccia, retrostante un accesso ad arcosolio, affiancata da analoga apertura di dimensioni minori e situata a un livello più basso, corrispondente con l'accesso a un pozzetto di raccolta, poco distinguibile in quanto intasato da detriti, o probabilmente a un tipo particolare di forno, aperto in fronte, con pancale a sigma per la cottura del pane, canale igneo o cinerario direttamente comunicanti con il piano di cottura, a un livello inferiore, come in un complesso polifunzionale di incerta destinazione, monastica o laica, presente nella valle di Zelve9 . In quest'ultimo esemplare la calotta del forno risulta appiattita e con una presa d'aria per il tiraggio del fumo decentrata e a forma di "becco di civetta"; il canale igneo è preceduto da un cinerario trasversale.

Nei casi esperiti, almeno tre nella valle di Zelve e in quella di Meskendir, l'ambiente del forno per la cottura del pane non appare chiuso in circolo, frontalmente, bensì aperto a semicerchio oltrepassato a sigma e con semicalotta tagliata verticalmente (figg. 28-29). A causa del moderno intasamento della vasca a cassone non è stato possibile definire se
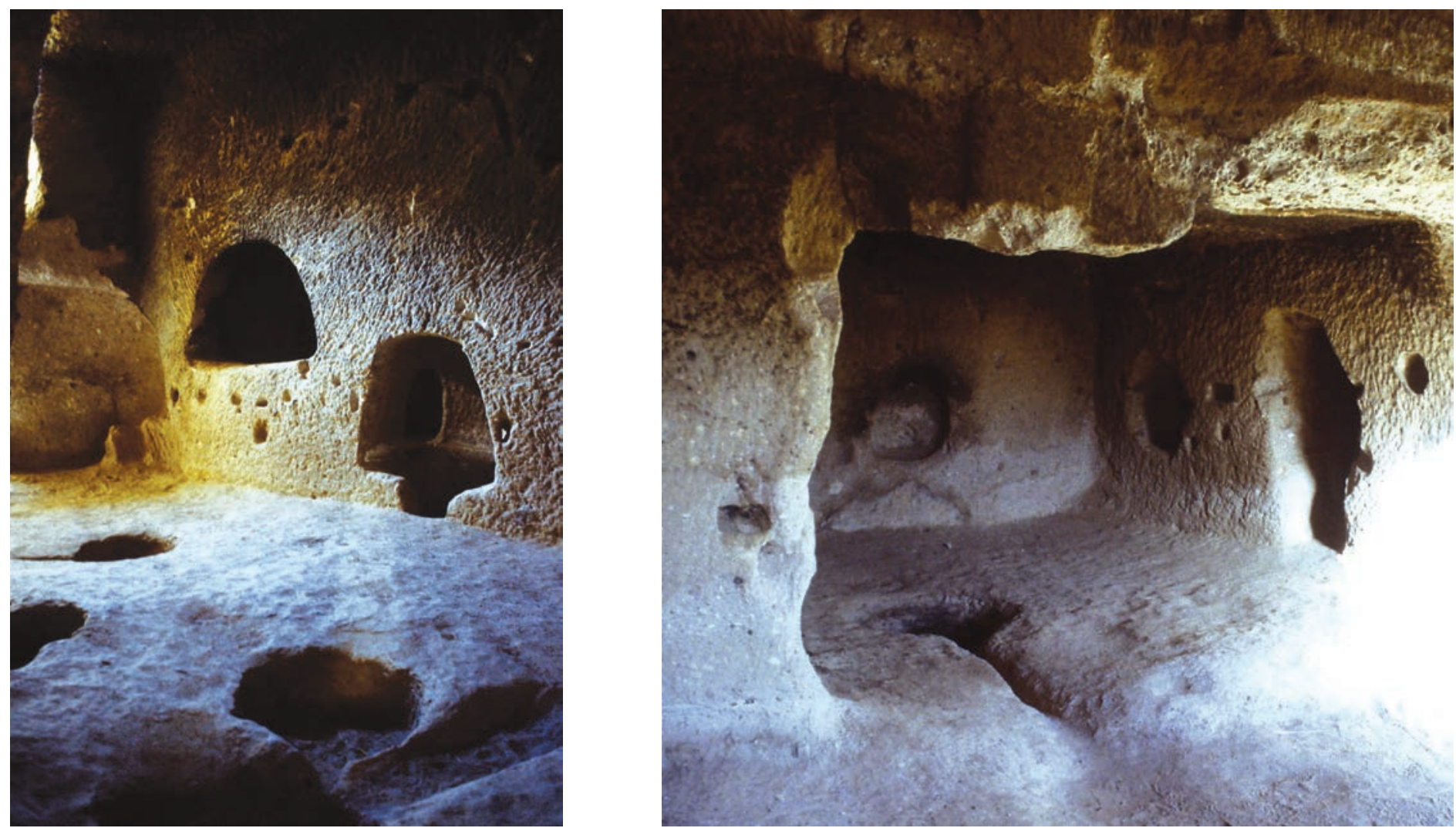

Figg. 26-27. Fornelli per brace scavati nel terreno: monastero A di Göreme e monastero di S. Sebastiano.

${ }^{8}$ Ibid., p. 479-48o.

${ }^{9}$ Ibid., p. $477-479$. 

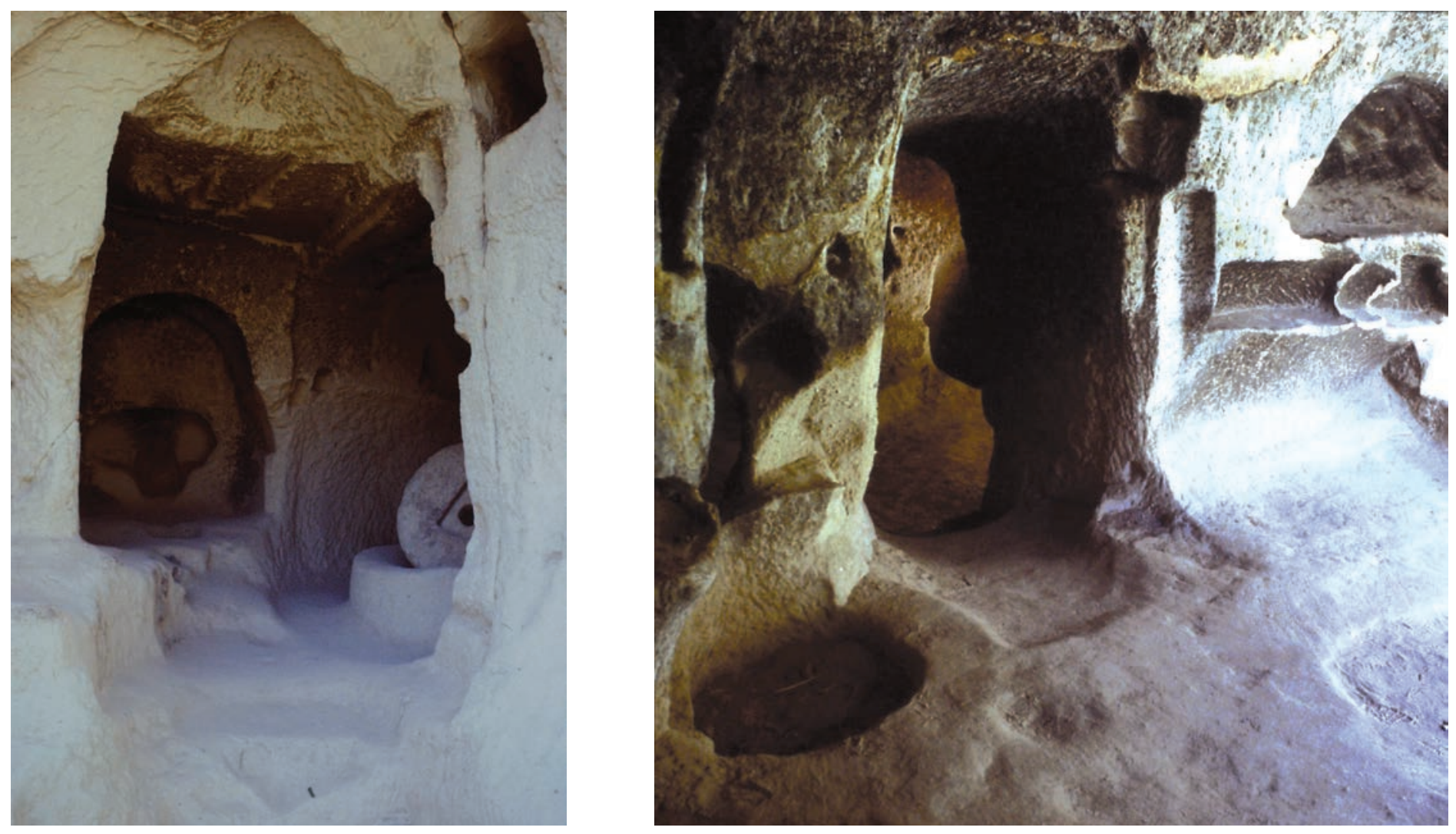

Figg. 28-29. Forni da pane nelle valli di Zelwe e Meskendir.
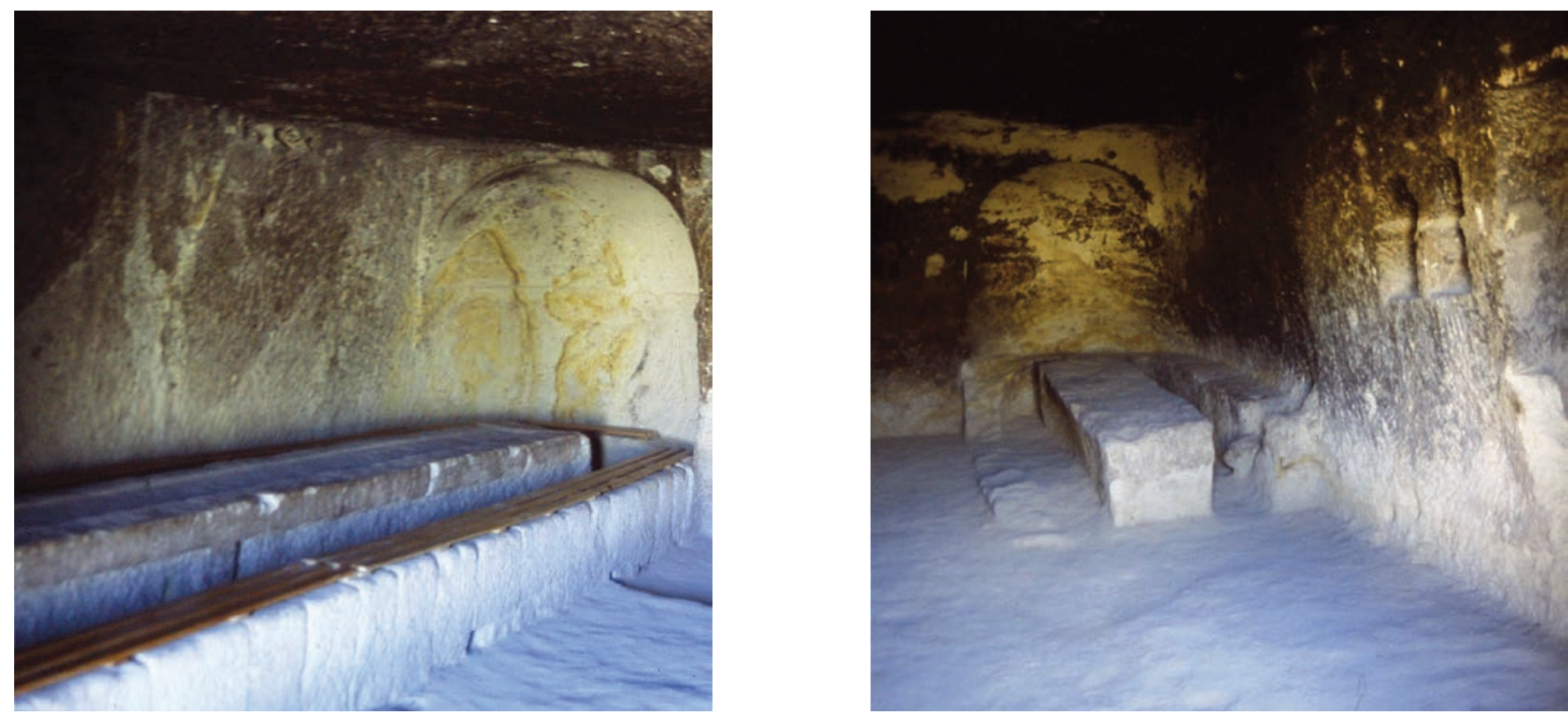

Figg. 30- 31. Refettori di due monasteri di Göreme.

l'invaso presente a Cemilköy fosse destinato ad acquaio per lavare frutta e verdura o per rigovernare le stoviglie, o ad altra funzione, come a tino per la spremitura dell'uva, non essendo stata accertata la profondità della vasca e la presenza o meno di un condotto di scarico dell'acqua nel pozzetto adiacente. Associati a questo genere di vasche troviamo frequentemente incavi per l'inserimento di tavole o di pali a seconda della loro forma, per il supporto di stoviglie o di oggetti imprecisati o per il sostegno di carrucole per attingere liquidi o sostenere materiali di forma e ampiezza consistenti.

I refettori, sempre separati dalle cucine, sono caratterizzati da una, o eccezionalmente due, come nel monastero degli Arcangeli di Cemilköy, lunghe strutture a bancone con doppia solcatura longitudinale rapportabili con sigma tricliniari di età tardoantica, ma fortemente allungate rispetto a esse ${ }^{10}$. Largo mediamente $2,20 \mathrm{~m}$, lungo variamente come quasi tutto l'ambiente destinato a refettorio e alto dal pavimento circa $60 \mathrm{~cm}$, il bancone, che aderisce a uno dei lati della sala, presenta una caratteristica doppia solcatura parallela cruciforme, larga dai 30 ai $40 \mathrm{~cm}$. Essa occupa tutta la lunghezza del bancone, terminando aperta e a fronte rettilinea, a un'estremità, curva a U, all'altra. Questa struttura, che si ripete pressoché identica in numerosi monasteri, come quelli della valle di Göreme e di Cemilköy (figg. 30-

${ }^{10}$ Ibid., p. 480 . 


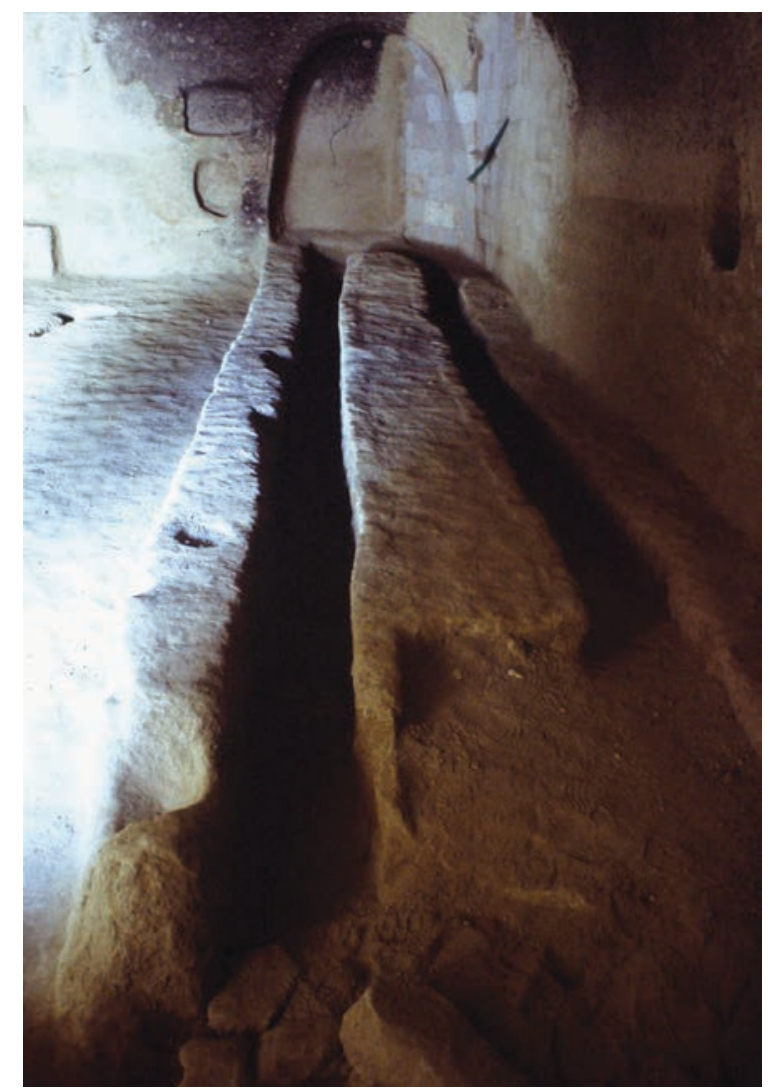

Fig. 32. Acquaio del refettorio di un monastero di Göreme.

31), costituisce la mensa, nel parallelepipedo centrale, a sezione rettangolare appiattita, la seduta dei monaci nei due murelli esterni contrapposti, a sezione rettangolare verticale, e l'incavo per i piedi dei commensali nella doppia solcatura parallela, a sezione rettangolare verticale. L'estremità lineare aperta costituisce la fronte di accesso alla mensa, quella curvilinea, sempre rivolta verso l'ingresso principale dell'ambiente e frequentemente enfatizzata da un sedile rialzato e/o da una nicchia ad arcosolio, come in un monastero della valle di Göreme, è destinata alla seduta dell'abate o del priore e dei suoi più stretti collaboratori. Nel monastero ora detto l'arcosolio è affiancato da un acquaio o lavabo per l'abluzione delle mani prima e dopo il pasto (fig. 32).

La nicchia rettangolare superiore era destinata a contenere un otre o una botticella per l'acqua, che attraverso un foro ricavato nel diaframma di roccia sottostante, versava, tramite un rubinetto di legno, in una nicchia di forma tondeggiante, a conchiglia, addetta al lavaggio delle mani. In altri monasteri di Göreme a fianco della nicchia del seggio del priore o dietro il sedile del lato lungo del bancone troviamo un altro arcosolio con vasca fungente da ampio lavabo o rinfrescatoio. Ancora poco chiara rimane l'interpretazione della o delle piccole nicchie ad arcosolio scavate nella parete situata alle spalle del lato lungo del bancone, ma sembra probabile, data la concavità della base, che contenessero acqua sussidiaria per le abluzioni delle mani dei commensali o fungessero da rinfrescatoio per le verdure o la frutta. Non presentando tracce di annerimento da fumo, è molto probabile che non dovessero ospitare lucerne o candele per l'illuminazione. Ma non dobbiamo generalizzare.

Al doppio bancone da mensa esistente nel monastero degli Arcangeli a Cemilköy conferisce un'indubbia monumentalità la parete divisoria risparmiata dalla escavazione della ignimbrite e traforata da diseguali archi a tutto sesto, sottolineati talvolta da solcature parallele, come quello destinato alla seduta del priore, e sostenuti da pilastri a sezione rettangolare (figg. 33-34). La presenza di uno o più fornelli sussidiari, scavati nel pavimento del refettorio per mantenere in caldo le vivande confezionate nelle cucine poco distanti, non sembra essere stata un fenomeno limitato ad alcuni monasteri della valle di Göreme.

\section{CONCLUSIONI}

La quantità e la densità dei fenomeni riscontrabili in Cappadocia, esponenzialmente superiore a quelle attestate in Occidente, attestano una precoce diffusione del monachesimo basiliano in questi territori e un'intensa presenza di forme d'insediamento rupestre nelle valli di erosione marginali rispetto alle città fondate in età imperiale, ma interessate da precedenti agglomerati urbani trogloditi mai completamente abbandonati.

Dalle indagini più recenti emergono paesaggi densamente strutturati dai monaci che scelsero questi siti per l'organizzazione delle loro attività spirituali, ma anche legate all'operosità positiva proposta e incoraggiata dai Padri della Chiesa. Come lo stesso Girolamo evocato all'inizio di questo intervento nel quale ci siamo limitati soltanto ad alcuni aspetti.
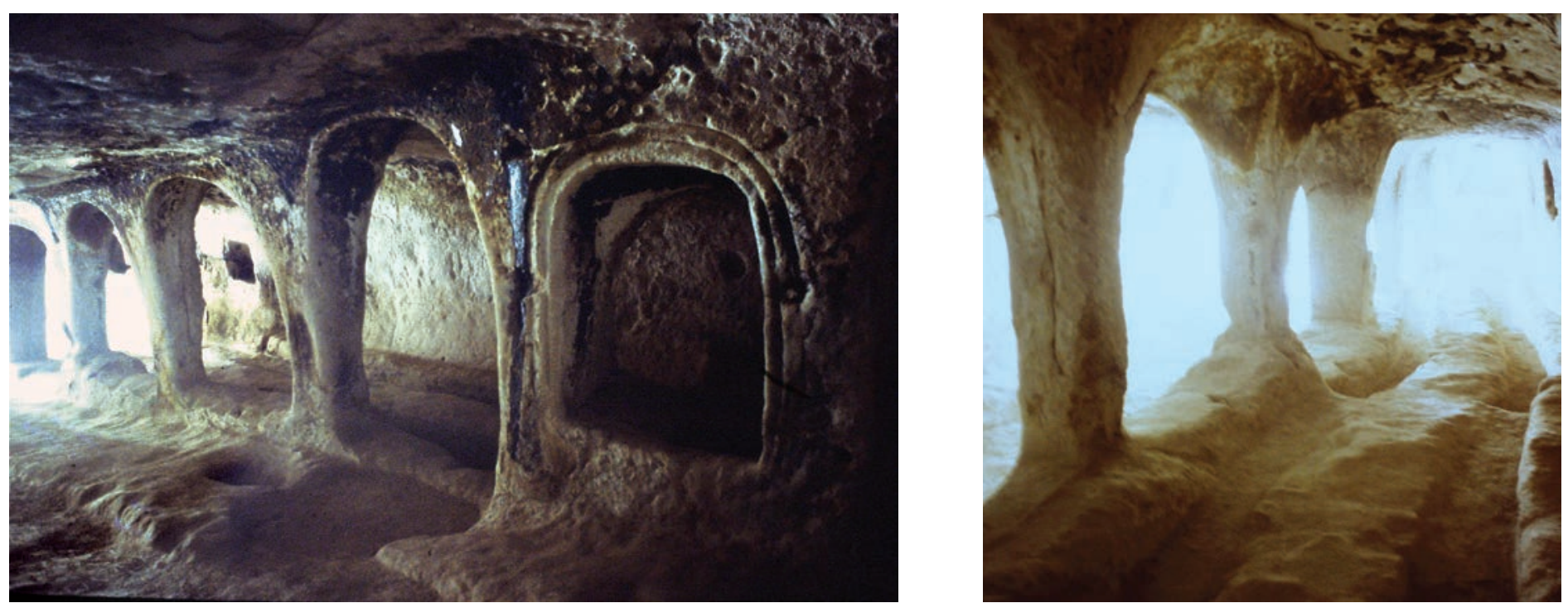

Figg. 33-34. Refettorio a doppia mensa nel monastero degli Arcengeli a Cemilköy. 